\title{
Demarcation Line Assessment in Anatomical Liver Resection: An Overview
}

Surgical Innovation

2020, Vol. 0(0) I-7

(C) The Author(s) 2020

Article reuse guidelines:

sagepub.com/journals-permissions DOI: 10.1 I $77 / 155335062095365$ | journals.sagepub.com/home/sri

(S)SAGE

\author{
Eric Felli, MSc ${ }^{1,2, *}$, Takeshi Urade, MD, PhD $^{1, *}$ (D), Mahdi \\ Al-Taher, MD', Emanuele Felli, MD ${ }^{3,4}$, Manuel Barberio, \\ MD, PhD $^{1,2}$, Laurent Goffin, MSc $^{5}$, Giuseppe M. Ettorre, \\ $M^{6}$, Jacques Marescaux, MD, FJSES(Hon), APSA ${ }^{1,7}$, \\ Patrick Pessaux, MD, PhD ${ }^{1,3,4}$, Lee Swanstrom, MD, \\ FASGE', and Michele Diana, MD, PhD ${ }^{1,2,3,7,8}$
}

\begin{abstract}
Anatomical liver resection (ALR) is the preferred oncological approach for the treatment of primary liver malignancies, such as hepatocellular carcinoma and intrahepatic cholangiocarcinoma. The demarcation line (DL) is formed by means of selective vascular occlusion and is used by surgeons to guide ALR. Emerging intraoperative technologies are playing a major role to enhance the surgeon's vision and ensure a precise oncologic surgery. In this article, a brief overview of modalities to assess the DL during ALRs is presented, from the established conventional techniques to future perspectives.
\end{abstract}

\section{Keywords}

anatomical liver resection, demarcation line, hepatectomy, hyperspectral imaging, indocyanine green

\section{Introduction}

Anatomical liver resection (ALR) is the preferred oncological approach for the treatment of primary liver malignancies, such as hepatocellular carcinoma (HCC) and intrahepatic cholangiocarcinoma. ${ }^{1-4}$ Although ALR is still controversial in the case of colorectal liver metastases, it may be beneficial to a subgroup of patients with RASmutated lesions. ${ }^{5}$ As the spreading of HCC follows portal branches, ALR is currently preferred because it entails wider resection margins and less remnant liver ischemia. ${ }^{6}$ It is indeed associated with better survival and disease-free survival as compared to patients who have not undergone ALR. ${ }^{1-4}$

The boundaries of the anatomical unit to be resected are defined according to the selective occlusion of its vascular supply. The consequent ischemia is visible on the liver surface and is delimited by a demarcation line (DL). The DL is the landmark that should be used intraoperatively in order to completely remove the ischemic region during ALRs. Recently, it has been reported that remnant liver ischemia is associated with worse long-term oncological outcomes, and venous congestion is associated with postoperative impaired liver function and liver regeneration. ${ }^{7-9}$ As a result, it is essential to accurately identify and monitor liver perfusion and the DL intraoperatively.
Recent progress in medical imaging technology, surgical technique, and perioperative management has greatly contributed to the safety and efficacy of surgery. ${ }^{10}$ This study aimed to provide an overview of established conventional techniques, new imaging technologies, and future perspectives, focusing on DL assessment in ALR (Table 1).

'IHU-Strasbourg, Institute of Image-Guided Surgery, France

${ }^{2}$ Institute of Physiology, EA3072 Mitochondria Respiration and Oxidative Stress, University of Strasbourg, France

${ }^{3}$ Department of General, Digestive, and Endocrine Surgery, University Hospital of Strasbourg, France

${ }^{4}$ INSERM UI I I0, Institute of Viral and Liver Disease, University of Strasbourg, France

${ }^{5}$ ICUBE/CNRS, University of Strasbourg, France

${ }^{6}$ Department of Transplantation and General Surgery, San Camillo Hospital, Italy

${ }^{7}$ IRCAD, Research Institute against Digestive Cancer, France

${ }^{8}$ ICUBE Laboratory, Photonic Instrumentation for Health, France

* These authors contributed equally to this work and share the position of first author.

\section{Corresponding Author:}

Takeshi Urade, IHU-Strasbourg, Institute of ImSage-Guided Surgery, I, place de l'Hôpital, Strasbourg 6709I, France.

Email: uradetl125@gmail.com 
Table I. Strong and Weak Points of Each Major Demarcation Technique.

\begin{tabular}{|c|c|c|}
\hline Demarcation Technique & Strong Point & Weak Point \\
\hline Direct visualization & $\begin{array}{l}\text { Basic technique to delineate the DL } \\
\text { No need for special medical device }\end{array}$ & $\begin{array}{l}\text { Operator-dependent } \\
\text { Occasional unclear DL due to the condition of the liver } \\
\text { parenchyma } \\
\text { Lack of perfusion quantification } \\
\text { Impossibility of visualizing the intrahepatic boundary }\end{array}$ \\
\hline Near-infrared imaging & $\begin{array}{l}\text { Excellent visualization of the DL } \\
\text { Possibility of visualizing the intrahepatic } \\
\text { boundary to some extent }\end{array}$ & $\begin{array}{l}\text { Impossibility of confirming the latest perfusion status due } \\
\text { to lasting fluorescence signals } \\
\text { Lack of perfusion quantification } \\
\text { Need for injection of indocyanine green }\end{array}$ \\
\hline $\begin{array}{l}\text { Augmented reality using 3D } \\
\text { virtual images }\end{array}$ & $\begin{array}{l}\text { Visualization of the predicted virtual DL } \\
\text { without vascular occlusion }\end{array}$ & $\begin{array}{l}\text { Positional accuracy error } \\
\text { Need for registration due to deformation of the liver and } \\
\text { organ motion } \\
\text { Time required for the construction of the } 3 D \text { virtual } \\
\text { image } \\
\text { Lack of perfusion quantification }\end{array}$ \\
\hline HYPER & $\begin{array}{l}\text { Excellent visualization of the DL } \\
\text { Perfusion quantification } \\
\text { Possibility of confirming the latest perfusion } \\
\text { status } \\
\text { No need for injection }\end{array}$ & $\begin{array}{l}\text { Need for the superimposition technique } \\
\text { Still not applied to intrahepatic boundary }\end{array}$ \\
\hline
\end{tabular}

Abbreviations: $\mathrm{DL}=$ demarcation line; $3 \mathrm{D}$ = 3-dimensional; $\mathrm{HYPER}=$ hyperspectral enhanced reality.
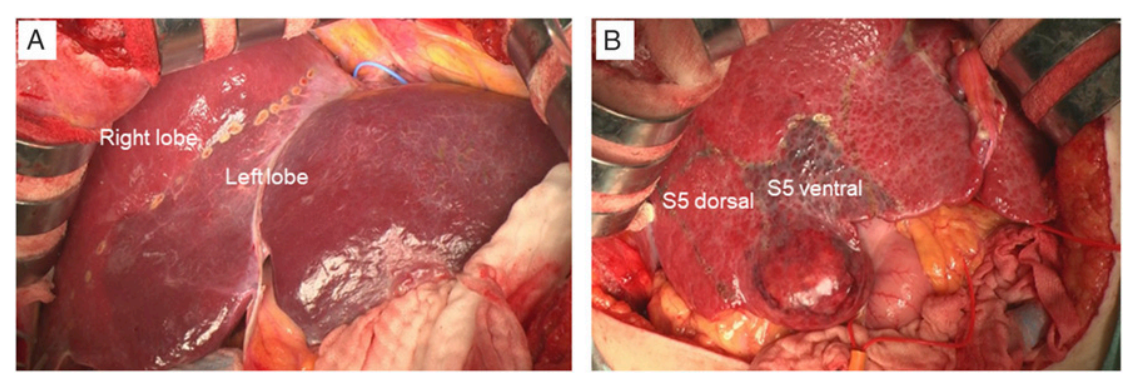

Figure I. Demarcation line via direct visualization on the liver surface in open liver surgery. (A) After ligation of the left hepatic artery and left portal vein branches in a left hepatectomy and (B) after portal staining of the ventral branch of liver segment $\mathrm{V}$ in segmentectomy $\mathrm{V}$.

\section{Direct Visualization on the Liver Surface}

The DL is generally identified by the surgeon's eye-based clinical evaluation. This can be achieved with either vascular occlusion or portal staining. The first approach consists in the occlusion of the vascular supply of the region of interest, either using selective portal and arterial branches or using the transection of the Glissonean pedicle as first described by Takasaki et al ${ }^{11}$ (Figure 1A). An additional monitoring of blood perfusion can be performed as described by Torzilli et $\mathrm{al}^{12}$ using an intraoperative ultrasound (IOUS)-guided vessel compression technique. The authors could demonstrate that this is a safe and effective approach to provide a demarcation assessment. The second approach consists in the application of portal staining, which is based on the direct injection of a visible dye, into the portal branch of the tumor-bearing segment (Figure 1B). Makuuchi et $\mathrm{al}^{13}$ proposed the puncture technique for portal staining, which is the most widely used approach to accomplish ALR. In this technique, the portal branch feeding the tumor to be resected is punctured under IOUS guidance, using a free-hand technique or with a tool to guide the puncture, and a dye (e.g., indigo carmine and methylene blue) is then injected into the vessel with temporary clamping of the hepatic artery at the hepatic pedicle. Once the stained region becomes evident on the liver surface, it is marked with electrocautery, creating a DL before liver resection. Additionally, Takayama et $\mathrm{al}^{14}$ have proposed a counterstaining technique, which can be used for all subsegments undergoing complete embolization and 
when the usual staining method is impossible because of the presence of arterioportal shunting or portal tumor thrombus.

Nowadays, the abovementioned techniques are considered standard approaches as basic conventional demarcation techniques are already applied for the curative treatment of HCC and other malignancies. ${ }^{11,13}$ However, during parenchymal transection, the inflow occlusion technique cannot recognize intersegmental/intersectional boundaries, and the reason is that hepatic veins and portal branches are used as anatomical landmarks to guide the resection. In addition, the portal staining technique cannot delineate the boundaries due to the dye wash out within a few minutes. In summary, conventional demarcation techniques in general have several drawbacks. They suffer from (1) being operator dependent, (2) the failing of the evaluation due to a nonclear demarcation, especially in patients with cirrhotic or steatotic livers, (3) the lack of quantification and characterization of the nonperfused section, and (4) the impossibility to recognize intersegmental/intersectional boundaries during parenchymal transection.

\section{Near-Infrared Imaging}

In the 1970s, protein-bound indocyanine green (ICG) was found to emit fluorescence peaking at about $840 \mathrm{~nm}$ under illumination with near-infrared (NIR) light (750$810 \mathrm{~nm}) .^{15}$ Because only a small quantity of light is absorbed by hemoglobin or water at this wavelength, fluorescence signals emitted by protein-bound ICG can be visualized through the connective tissue, which is 5- to 10mm thick. ${ }^{16}$ ICG fluorescence imaging has been used as an intraoperative navigation tool to visualize lymphatic flow and/or blood flow in various digestive surgeries, ${ }^{17,18}$ vascular surgery, and neurosurgery. In liver surgery, for the first time, Aoki et $\mathrm{al}^{19}$ reported that ICG fluorescence imaging was a safe and reproducible intraoperative technique to identify the hepatic segment and subsegment for ALR. As a result, it can be applied to identify the DL through the visualization of parenchymal perfusion in both open and laparoscopic ALR (Figure 2). There are 2 staining methods to identify anatomical liver regions with ICG. A positive staining method is performed to detect anatomical liver regions via the injection of ICG into the portal branches under IOUS guidance, ${ }^{19-23}$ and a negative staining method is performed to detect anatomical liver regions as nonfluorescing regions via the intravenous injection of ICG after clamping portal branches. ${ }^{20,24-27}$ Fluorescence imaging can offer an improvement over gross examinations based on conventional dye staining. ${ }^{22}$ In addition, our team has reported the use of an interventional radiology-assisted selective intra-arterial injection technique for positive staining. ${ }^{28}$ This novel technique may be applied for complicated surgical procedures, such as laparoscopic segmentectomy V, VI, VII, or VIII. ${ }^{29}$

In open surgery, ICG fluorescence imaging requires the dimming of light in the operative field to prevent white light contamination. It also requires that surgeons shift their field of vision to and from the images and real organs under a darkened operative field. To address such issues, a new medical imaging system, which uses an ICG emission signal and an active projection mapping, was developed by Nishino et al. ${ }^{30}$ Several studies reported that intersegmental/sectional boundaries between fluorescing and nonfluorescing parenchyma could be recognized to some extent during parenchymal transection. ${ }^{27,29,31}$ These boundaries are useful landmarks to recognize the intrahepatic direction and guide the transection of the liver parenchyma. However, further studies are required to prove whether the boundaries are accurate because ICG fluorescence could possibly be visualized through 5-10 $\mathrm{mm}$ of deeper parenchyma.

The major drawback with ICG fluorescence imaging lies in the fact that, once ICG has been administered, the nonischemic parenchyma emits fluorescent signals, which last for hours. This long-lasting fluorescence signal makes it impossible to assess the latest status of perfusion. In fact, the ischemic area or congested area, which can occur incidentally, could be left in the remnant liver after transection.

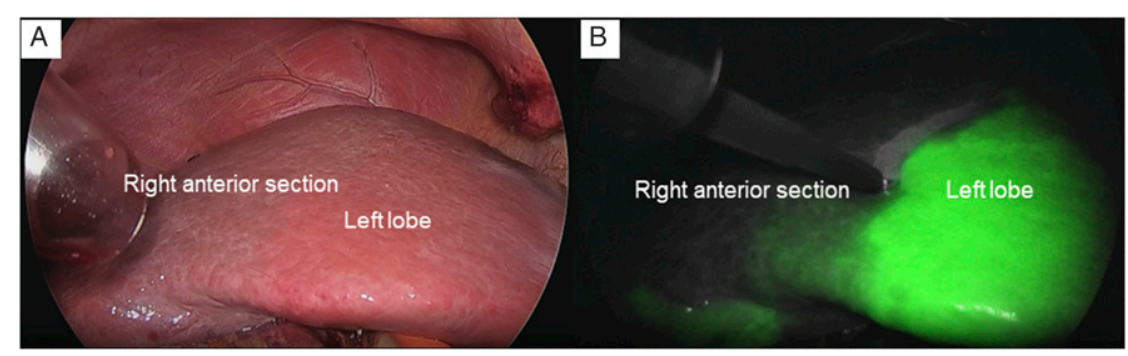

Figure 2. Demarcation line after ligation of the right anterior Glissonean pedicle in a laparoscopic right anterior sectionectomy. (A) Normal laparoscopic image and (B) indocyanine green fluorescence imaging. 
Several preclinical NIR fluorophores are currently under evaluation for clinical translation. One of these fluorophores is the IRDye ${ }^{\circledR} 800 \mathrm{CW}$ dye (excitation: $778 \mathrm{~nm}$ and emission: $794 \mathrm{~nm})$. The IRDye ${ }^{\circledR} 800 \mathrm{CW}$ dye was evaluated with protein/antibody labeling applications such as bevacizumab $^{32,33}$ and cetuximab. ${ }^{34,35}$ Qian et $\mathrm{al}^{36}$ studied this dye for liver resection guided by means of fluorescence in a mouse liver model and a pig liver model. Human monocytes were labeled with the IRDye ${ }^{\circledR}$ $800 \mathrm{CW}$ dye, which resulted in an excellent liver segment boundary demarcation in isolated mouse and pig perfusion and via intraportal injection in mouse liver tumor models. This is a promising finding, which has the potential to aid in surgical navigation during liver surgery.

\section{Augmented Reality Using Preoperative 3-Dimensional Virtual Images}

With the development of medical imaging and innovative computer programs, preoperative 3-dimensional (3D) virtual images created from computed tomography or magnetic resonance imaging can be used to understand the spatial relationship between tumors and vasculatures and to predict the resected and remnant liver volume for a precise and safe liver surgery. Additionally, the use of augmented reality (AR) technology which is the superimposition of virtual images onto real-time patient images allowed us to identify the anatomy of the intrahepatic and extrahepatic structures on the same monitor during the operation. It provides an enhanced virtual reality rendering to give the surgeon essential information to optimize navigation during complex surgery and to reduce intraoperative and postoperative complications. ${ }^{37}$ Since our team reported the first use of the AR guidance technique for general surgery, ${ }^{38}$ it has been applied to various operations, including liver surgery. AR using 3D virtual images reconstructed from the preoperative computed tomography data set was reported not only in open liver surgery ${ }^{39-41}$ but also for minimally invasive liver resection by our team. ${ }^{42-44}$ When a segmented preoperative $3 \mathrm{D}$ virtual image for ALR is superimposed onto the patient's operative field image, the predicted virtual DL can be visualized on the liver without vascular occlusion (Figure 3), and intrahepatic boundaries can also be visualized. Consequently, it may contribute to safe parenchymal transection without vascular injury and excessive resection of the liver in ALR.

The main limitation of AR is the use of a fixed virtual model, which is always influenced by liver deformation, heartbeat, and respiratory motion. As a result, intraoperative AR accuracy is limited in the event of fusion with more mobile or deformable structures. The current registration process involves some degrees of interaction with computer scientists to reduce the positional accuracy

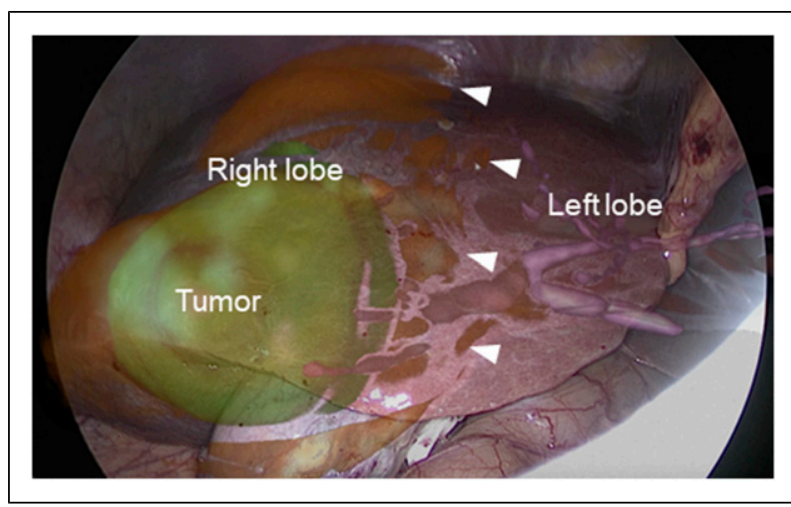

Figure 3. Demarcation line (arrow heads) by means of superimposition of preoperative computed tomography simulation image during laparoscopic right hepatectomy.

error because automatic registration is challenging and represents a field of ongoing experimental research. The main goal of the future in this research field is to develop a fully automatic registration system with precise organ motion prediction.

\section{HYPerspectral Enhanced Reality}

Alternative imaging technologies that result in a contrastfree, stronger demarcation of segmental boundaries may improve the surgical procedure and its outcomes. The opportunity to quantify and identify the DL in a noninvasive way, with the overall image mapping in real time, would be ideal to guide ALRs. Hyperspectral imaging (HSI) is an optical imaging technique, which allows a real-time perfusion analysis of the tissue. It combines a photo camera with a spectroscope to allow for the analysis of relative absorbance at different depths and to provide relative oxygenation of the organ tissue. It is also possible to quantify the superficial tissue oxygen saturation (StO2) at a depth of $\sim 1 \mathrm{~mm}$ and the oxygenated hemoglobin quantification within the NIR spectrum at a depth of 4-6 mm. ${ }^{45}$ HSI has been evaluated in several medical applications, ${ }^{46}$ including cancer tissue discrimination, ${ }^{47,48}$ gastrointestinal anastomosis perfusion, ${ }^{49-51}$ and anatomical structures identification. ${ }^{52,53}$ However, the lack of a video rate with current devices prevents a smooth application as a surgical navigation tool due to the static side-by-side image display. To overcome this limitation, our group has developed and experimentally validated the concept of HYPerspectral-based enhanced reality (HYPER), in a model of bowel surgery. ${ }^{54}$ HYPER overlays images generated by HSI with real-time videos of the surgical field, providing a precise AR environment visible to the surgeon on a screen. Importantly, the HSI NIR index can quantify and image tissue perfusion more precisely than the human eye. Our group has recently validated HYPER as a useful and effective tool to provide 

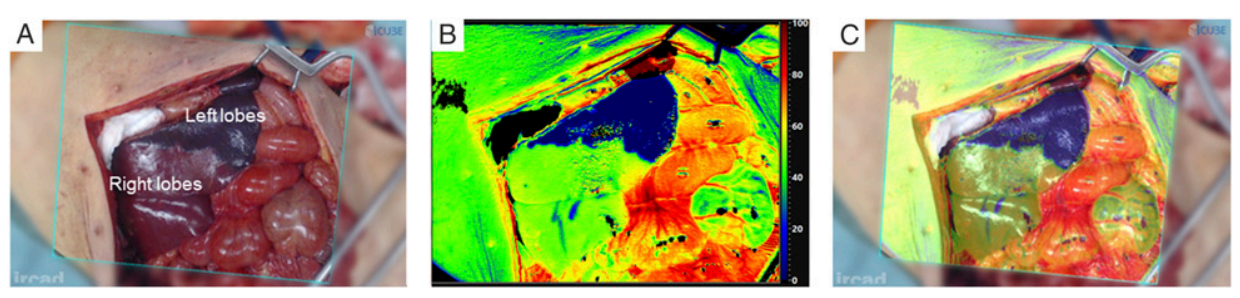

Figure 4. Demarcation line after the ligation of the left hepatic pedicle branches in an open left hepatectomy in a porcine model. (A) RGB camera image, (B) near-infrared image, and (C) hyperspectral-based enhanced reality, near-infrared image superimposed onto the RGB video. Abbreviations: RGB, red/green/blue.

guidance during $\mathrm{ALRs}^{55}$ (Figure 4). The HYPER DL assessment was performed experimentally using an inflow clamping method, which allowed an effective discrimination of the boundary between perfused and nonperfused regions. From our initial experiences, it is shown that HYPER is a promising technology for image-guided surgery including the advantages of providing quantitative data on perfusion in real time and without the need for dye injection. From the initial proof of concept of HYPER applied to the DL assessment, we are moving toward the evaluation of the whole resection plane. Additionally, we need to evaluate the combined HYPER technique with a portal staining method to appreciate the interplane perfusion because there will be different spectrums between blood and dyes theoretically. The downside is that the superimposition of HSI and red/green/blue video images is not yet directly integrated into the hyperspectral camera, and for that reason, the overlay requires a few extra seconds. Nowadays, the newest generations of hyperspectral cameras can provide images at a rapid pace but not at an acceptable video rate (frames/seconds). Irrespective of its limitations, HYPER represents a good intraoperative real-time noninvasive tool, which can provide an overall mapping to guide surgeons and help them understand the latest evaluation of liver perfusion.

\section{Conclusions}

New technologies for demarcation assessment during liver ALRs aim to provide more information to surgeons in order to standardize the surgical approach by means of quantification and characterization of liver perfusion. NIR imaging is an appropriate and effective tool for liver ALRs. AR using $3 \mathrm{D}$ virtual images helps to visualize the virtual DL obtained by means of preoperative imaging data set segmentation under accurate registration. HSI yields a quantifiable measurement of the liver tissue perfusion and displays a precise DL to guide the surgical dissection.

\section{Acknowledgments}

We would like to thank Guy Temporal and Christopher Burel for their assistance with medical English proofreading.

\section{Author Contributions}

Study concept and design: Eric Felli, Takeshi Urade, and Michele Diana.

Acquisition of data: Eric Felli, Takeshi Urade, Mahdi Al-Taher, Emanuele Felli, Manuel Barberio, Laurent Goffin, and Patrick Pessaux.

Analysis and interpretation of data: Eric Felli, Takeshi Urade, and Mahdi Al-Taher.

Study supervision: Giuseppe Maria Ettorre, Jacques Marescaux, Patrick Pessaux, Lee Swanström, and Michele Diana.

\section{Declaration of Conflicting Interests}

The authors declared no potential conflicts of interest with respect to the research, authorship, and/or publication of this article.

\section{Funding}

The authors disclosed receipt of the following financial support for the research, authorship, and/or publication of this article: This work was partly funded by the ARC Foundation for Cancer Research (9, rue Guy Môquet - BP 90003 - 94803 Villejuif Cedex-France) via the ELIOS grant (PI Michele Diana).

\section{ORCID iDs}

Takeshi Urade (D) https://orcid.org/0000-0002-4557-1777 Michele Diana (D) https://orcid.org/0000-0002-1390-8486

\section{References}

1. Chen J, Huang K, Wu J, et al. Survival after anatomic resection versus nonanatomic resection for hepatocellular carcinoma: A meta-analysis. Dig Dis Sci. 2011;56: 1626-1633.

2. Zhou Y, Xu D, Wu L, Li B. Meta-analysis of anatomic resection versus nonanatomic resection for hepatocellular carcinoma. Langenbecks Arch Surg. 2011;396: 1109-1117.

3. Tan Y, Zhang W, Jiang L, Yang J, Yan L. Efficacy and safety of anatomic resection versus nonanatomic resection in patients with hepatocellular carcinoma: A systemic review and meta-analysis. PLoS One. 2017;12:e0186930.

4. Si A, Li J, Yang Z, et al. Impact of anatomical versus nonanatomical liver resection on short- and long-term outcomes for patients with intrahepatic cholangiocarcinoma. Ann Surg Oncol. 2019;26:1841-1850. 
5. Margonis GA, Buettner S, Andreatos N, et al. Anatomical resections improve disease-free survival in patients with KRAS-mutated colorectal liver metastases. Ann Surg. 2017; 266:641-649.

6. Hasegawa K, Kokudo N, Imamura H, et al. Prognostic impact of anatomic resection for hepatocellular carcinoma. Ann Surg. 2005;242:252-259.

7. Cho JY, Han H-S, Choi Y, et al. Association of remnant liver ischemia with early recurrence and poor survival after liver resection in patients with hepatocellular carcinoma. JAMA Surgery. 2017;152:386-392.

8. Yamashita S, Venkatesan AM, Mizuno T, et al. Remnant liver ischemia as a prognostic factor for cancer-specific survival after resection of colorectal liver metastases. JAMA Surg. 2017;152:e172986.

9. Scatton O, Plasse M, Dondero F, Vilgrain V, Sauvanet A, Belghiti J. Impact of localized congestion related to venous deprivation after hepatectomy. Surgery. 2008;143:483-489.

10. Mascagni P, Longo F, Barberio M, et al. New intraoperative imaging technologies: Innovating the surgeon's eye toward surgical precision. J Surg Oncol. 2018;118:265-282.

11. Takasaki K. Glissonean pedicle transection method for hepatic resection: A new concept of liver segmentation. J Hepato-Biliary-Pancreatic Surg. 1998;5:286-291.

12. Torzilli G, Procopio F, Cimino M, et al. Anatomical segmental and subsegmental resection of the liver for hepatocellular carcinoma: A new approach by means of ultrasound-guided vessel compression. Ann Surg. 2010; 251:229-235.

13. Makuuchi M, Hasegawa H, Yamazaki S. Ultrasonically guided subsegmentectomy. Surg Gynecol Obstet. 1985;161: 346-350.

14. Takayama T, Makuuchi M, Watanabe K, et al. A new method for mapping hepatic subsegment: Counterstaining identification technique. Surgery. 1991;109:226-229.

15. Landsman ML, Kwant G, Mook GA, Zijlstra WG. Lightabsorbing properties, stability, and spectral stabilization of indocyanine green. J Appl Physiol. 1976;40:575-583.

16. Ishizawa T, Saiura A, Kokudo N. Clinical application of indocyanine green-fluorescence imaging during hepatectomy. Hepatobiliary Surg Nutr. 2016;5:322-328.

17. van Manen L, Handgraaf HJM, Diana M, et al. A practical guide for the use of indocyanine green and methylene blue in fluorescence-guided abdominal surgery. J Surg Oncol. 2018;118:283-300.

18. Baiocchi GL, Diana M, Boni L. Indocyanine green-based fluorescence imaging in visceral and hepatobiliary and pancreatic surgery: State of the art and future directions. World J Gastroenterol. 2018;24:2921-2930.

19. Aoki T, Yasuda D, Shimizu Y, et al. Image-guided liver mapping using fluorescence navigation system with indocyanine green for anatomical hepatic resection. World $J$ Surg. 2008;32:1763-1767.

20. Ishizawa T, Zuker NB, Kokudo N, Gayet B. Positive and negative staining of hepatic segments by use of fluorescent imaging techniques during laparoscopic hepatectomy. Arch Surg. 2012;147:393-394.

21. Sakoda M, Ueno S, Iino S, et al. Anatomical laparoscopic hepatectomy for hepatocellular carcinoma using indocyanine green fluorescence imaging. J Laparoendosc Adv Surg Tech. 2014;24:878-882.

22. Miyata A, Ishizawa T, Tani K, et al. Reappraisal of a dyestaining technique for anatomic hepatectomy by the concomitant use of indocyanine green fluorescence imaging. $J$ Am Coll Surg. 2015;221:e27-e36.

23. Aoki T, Koizumi T, Mansour DA, et al. Ultrasound-guided preoperative positive percutaneous indocyanine green fluorescence staining for laparoscopic anatomical liver resection. J Am Coll Surg. 2020;230:e7-e12.

24. Mizuno T, Sheth R, Yamamoto M, et al. Laparoscopic glissonean pedicle transection (Takasaki) for negative fluorescent counterstaining of segment 6. Ann Surg Oncol. 2017;24:1046-1047.

25. Terasawa M, Ishizawa T, Mise $\mathrm{Y}$, et al. Applications of fusion-fluorescence imaging using indocyanine green in laparoscopic hepatectomy. Surg Endosc. 2017;31: 5111-5118.

26. Nomi T, Hokuto D, Yoshikawa T, Matsuo Y, Sho M. A novel navigation for laparoscopic anatomic liver resection using indocyanine green fluorescence. Ann Surg Oncol. 2018;25:3982.

27. Urade T, Sawa H, Iwatani Y, et al. Laparoscopic anatomical liver resection using indocyanine green fluorescence imaging. Asian J Surg. 2020;43:362-368.

28. Diana M, Liu Y-Y, Pop R, et al. Superselective intra-arterial hepatic injection of indocyanine green (ICG) for fluorescence image-guided segmental positive staining: Experimental proof of the concept. Surg Endosc. 2017;31: 1451-1460.

29. Ueno M, Hayami S, Sonomura T, et al. Indocyanine green fluorescence imaging techniques and interventional radiology during laparoscopic anatomical liver resection (with video). Surg Endosc. 2018;32:1051-1055.

30. Nishino H, Hatano E, Seo S, et al. Real-time navigation for liver surgery using projection mapping with indocyanine green fluorescence. Ann Surg. 2018;267:1134-1140.

31. Inoue Y, Arita J, Sakamoto T, et al. Anatomical liver resections guided by 3-dimensional parenchymal staining using fusion indocyanine green fluorescence imaging. Ann Surg. 2015;262:105-111.

32. de Jongh SJ, Tjalma JJJ, Koller M, et al. Back-table fluorescence-guided imaging for circumferential resection margin evaluation using bevacizumab- $800 \mathrm{CW}$ in patients with locally advanced rectal cancer. $J$ Nucl Med. 2019;61: 655-661. doi:10.2967/jnumed.119.232355

33. Ter Weele EJ, Terwisscha van Scheltinga AGT, Linssen $\mathrm{MD}$, et al. Development, preclinical safety, formulation, and stability of clinical grade bevacizumab- $800 \mathrm{CW}$, a new near infrared fluorescent imaging agent for first in human use. Eur J Pharm Biopharm. 2016;104:226-234.

34. Tummers WS, Miller SE, Teraphongphom NT, et al. Intraoperative pancreatic cancer detection using tumorspecific multimodality molecular imaging. Ann Surg Oncol. 2018;25:1880-1888.

35. Tummers WS, Miller SE, Teraphongphom NT, et al. Detection of visually occult metastatic lymph nodes using molecularly targeted fluorescent imaging during surgical resection of pancreatic cancer. HPB. 2019;21:883-890. 
36. Qian B, Kyuno D, Schäfer M, Gross W, Mehrabi A, Ryschich E. Liver segment imaging using monocyte sequestration: A potential tool for fluorescence-guided liver surgery. Theranostics. 2018;8:6101-6110.

37. Quero G, Lapergola A, Soler L, et al. Virtual and augmented reality in oncologic liver surgery. Surg Oncol Clin N Am. 2019;28:31-44.

38. Marescaux J, Rubino F, Arenas M, Mutter D, Soler L. Augmented-reality-assisted laparoscopic adrenalectomy. JAMA. 2004;292:2014-2015.

39. Okamoto T, Onda S, Matsumoto M, et al. Utility of augmented reality system in hepatobiliary surgery. $J$ HepatoBiliary-Pancreat Sci. 2013;20:249-253.

40. Ntourakis D, Memeo R, Soler L, Marescaux J, Mutter D, Pessaux P. Augmented reality guidance for the resection of missing colorectal liver metastases: An initial experience. World J Surg. 2016;40:419-426.

41. Golse N, Petit A, Lewin M, Vibert E, Cotin S. Augmented reality during open liver surgery using a markerless nonrigid registration system. J Gastrointest Surg. 2020. doi:10. 1007/s11605-020-04519-4

42. Soler L, Nicolau S, Pessaux P, Mutter D, Marescaux J. Realtime 3D image reconstruction guidance in liver resection surgery. Hepatobiliary Surg Nutr. 2014;3:73-81.

43. Pessaux P, Diana M, Soler L, Piardi T, Mutter D, Marescaux J. Towards cybernetic surgery: Robotic and augmented reality-assisted liver segmentectomy. Langenbeck's Arch Surg. 2015;400:381-385.

44. Hallet J, Soler L, Diana M, et al. Trans-thoracic minimally invasive liver resection guided by augmented reality. $\mathrm{J} \mathrm{Am}$ Coll Surg. 2015;220:e55-60.

45. Holmer A, Marotz J, Wahl P, Dau M, Kämmerer PW. Hyperspectral imaging in perfusion and wound diagnostics methods and algorithms for the determination of tissue parameters. Biomed Eng/Biomed Tech. 2018;63:547-556.
46. Lu G, Fei B. Medical hyperspectral imaging: A review. J Biomed Optic. 2014;19:010901.

47. Akbari H, Uto K, Kosugi Y, Kojima K, Tanaka N. Cancer detection using infrared hyperspectral imaging. Canc Sci. 2011;102:852-857.

48. Martinez B, Leon R, Fabelo H, et al. Most relevant spectral bands identification for brain cancer detection using hyperspectral imaging. Sensors. 2019;19:5481. doi:10.3390/ s19245481

49. Köhler H, Jansen-Winkeln B, Maktabi M, et al. Evaluation of hyperspectral imaging (HSI) for the measurement of ischemic conditioning effects of the gastric conduit during esophagectomy. Surg Endosc. 2019;33:3775-3782.

50. Jansen-Winkeln B, Holfert N, Köhler H, et al. Determination of the transection margin during colorectal resection with hyperspectral imaging (HSI). Int J Colorectal Dis. 2019;34:731-739.

51. Jansen-Winkeln B, Maktabi M, Takoh JP, et al. Hyperspektral-imaging bei gastrointestinalen anastomosen. Chirurg. 2018;89:717-725. doi:10.1007/s00104-0180633-2

52. Barberio M, Maktabi M, Gockel I, et al. Hyperspectral based discrimination of thyroid and parathyroid during surgery. Cur Dir Biomed Eng. 2018;4:399-402. doi:10. 1515/cdbme-2018-0095

53. Zuzak KJ, Naik SC, Alexandrakis G, Hawkins D, Behbehani $\mathrm{K}$, Livingston $\mathrm{E}$. Intraoperative bile duct visualization using near-infrared hyperspectral video imaging. Am J Surg. 2008; 195:491-497.

54. Barberio M, Longo F, Fiorillo C, et al. HYPerspectral enhanced reality (HYPER): A physiology-based surgical guidance tool. Surg Endosc. 2020;34:1736-1744.

55. Urade T, Felli E, Barberio M, et al. Hyperspectral enhanced reality (HYPER) for anatomical liver resection. Surg Endosc. 2020. doi:10.1007/s00464-020-07586-5 